\title{
Fertilité des rameaux anticipés de vigne (Vitis vinifera L.). I. Expression au vignoble
}

\author{
Chantal OLIVAIN \& Roger BESSIS \\ Université de Bourgogne, Laboratoire des Sciences de la vigne, Faculté des Sciences Mirande, B.P. 138, \\ F 21004 Dijon Cedex
}

RÉSUMÉ La fertilité des rameaux anticipés du cépage Pinot est étudiée sur sarments écimés et non écimés. Sur sarments
non écimés, la fertilité des rameaux anticipés est toujours très faible. Sur sarments écimés, l'obtention et le
maintien d'une fertilité maximale sont fonction de l'importance et de l'époque d'écimage. L'intensité de cette
fertilité est en relation avec le rang d'insertion des rameaux anticipés sur le sarment.

Mots clés additionnels : Ecimage.

The fertility of grapevine lateral shoots (cv. Pinot) was investigated with decapitated and non-decapitated shoots. On non-decapitated shoots, lateral-shoot fertility was always low. On decapitated shoots, obtaining and maintaining maximum fertility depended on the degree and the date of decapitation. Fertility intensity varied with the position of the lateral shoots on the primary shoot.

Additional key words : Decapitation.

\section{INTRODUCTION}

Chez Vitis vinifera L. cépage Pinot, les caractères de la fertilité des rameaux anticipés sont très mal connus. D'une manière générale, on sait que des grappes sont présentes sur les rameaux anticipés qui se développent après les divers rognages pratiqués au vignoble. Dans une précédente note (OLIVAIN \& BESSIS, 1987), nous avons étudié, sur sarments non écimés, l'organogenèse inflorescentielle dans les bourgeons anticipés. La présente étude concerne le devenir des inflorescences formées. Le but est de caractériser, sur sarments écimés et non écimés, l'expression de la fertilité des rameaux anticipés c'est-à-dire définir les conditions qui conduisent au plein développement des inflorescences ou à leur avortement.

\section{MATÉRIEL ET MÉTHODES}

Tous les essais sont conduits sur cépage Pinot, dans le vignoble expérimental de l'Université de Bourgogne situé à Marsannay-la-Côte. Les expériences ont été répétées sur 4 années $(1978,1979,1980,1982)$ et ont montré une bonne reproductibilité ; en conséquence, les résultats chiffrés exposés sont essentiellement tirés de l'expérimentation 1982. Nous utiliserons le terme " anticipé » pour désigner le bourgeon anticipé (prompt bourgeon) ainsi que le rameau anticipé (« entre-cœur », « rebiot», « lateral-shoot »). Les expériences sont effectuées sur les anticipés des rangs 3, 6, 10 et 15 (numération à partir de la base).

Nous avons cherché à connaître l'expression de la fertilité des anticipés qui se développent à la suite de différents écimages. Un protocole de décapitation est donc appliqué à chacun des rangs testés depuis la mimai jusqu'à la fin juillet. Sur une pousse de vigne, le méristème terminal forme avec les très jeunes feuilles qui le recouvrent un sommet végétatif d'environ $5 \mathrm{~mm}$; le premier entre-nœud visible sous cet apex mesure de 1 à $2 \mathrm{~mm}$ et l'apex peut être facilement détaché à l'aide d'une pince. Cet écimage est notre écimage minimal de référence appelé « apex », les écimages ultérieurs sont codifiés par le nombre de nœuds et de feuilles enlevés en plus de l'apex (type d'écimage, tabl. 1). Pour un rang déterminé, l'importance de l'écimage augmente donc en fonction de l'époque d'écimage et l'anticipé situé immédiatement sous la décapitation est de plus en plus organisé. L'importance de l'écimage est indiquée par la longueur approximative de la partie supprimée. L'état d'organisation de l'anticipé au moment de l'écimage est évalué 
TABLEAU 1

Caractéristiques des écimages effectués aux rangs 6 et 10 lors de l'année 1982.

Characteristics of decapitation carried out during 1982 (nodes 6 and 10 ).

\begin{tabular}{|c|c|c|c|c|}
\hline \multirow{2}{*}{ Type d'écimage } & \multirow{2}{*}{$\begin{array}{l}\text { Longueur } \\
\text { approximative } \\
\text { de l'écimage } \\
\text { (cm) }\end{array}$} & \multirow{2}{*}{$\begin{array}{l}\text { Situation } \\
\text { de l'anticipé } \\
\text { par rapport } \\
\text { à l'apex }\end{array}$} & \multicolumn{2}{|c|}{$\begin{array}{c}\text { Epoque } \\
\text { d'écimage }\end{array}$} \\
\hline & & & rang 6 & rang 10 \\
\hline apex & $0,3-0,5$ & 1 & $11 / 5$ & $21 / 5$ \\
\hline apex +1 feuille & $0,5-0,7$ & 2 & $13 / 5$ & $24 / 5$ \\
\hline apex +2 feuilles & $0,8-1$ & 3 & $15 / 5$ & $27 / 5$ \\
\hline apex +3 feuilles & $1,5-2,5$ & 4 & $17 / 5$ & $31 / 5$ \\
\hline apex +5 feuilles & $6-7,5$ & 6 & $21 / 5$ & $4 / 6$ \\
\hline apex +7 feuilles & $20-25$ & 8 & $27 / 5$ & $9 / 6$ \\
\hline apex +9 feuilles & $40-45$ & 10 & $3 / 6$ & $14 / 6$ \\
\hline apex +11 feuilles & $60-70$ & 12 & $9 / 6$ & $18 / 6$ \\
\hline apex +14 feuilles & $80-90$ & 15 & $14 / 6$ & $23 / 6$ \\
\hline apex +19 feuilles & $120-140$ & 20 & $29 / 6$ & $8 / 7$ \\
\hline
\end{tabular}

d'après sa position par rapport à l'apex ; par exemple, pour l'écimage « apex » (fig. 1) pratiqué au-dessus du nœud 6, l'anticipé étudié est inséré au premier nœud sous l'apex, il est également à l'aisselle de la $1^{\text {re }}$ feuille qui se détache partiellement du sommet végétatif ; il est alors désigné par le chiffre 1. L'importance et l'époque d'écimage ainsi que la situation des anticipés par rapport à l'apex sont reportées au tableau 1 pour les rangs 6 et 10 lors de l'année 1982. Les premiers écimages libèrent des anticipés à l'aisselle de jeunes feuilles en croissance tandis que les derniers écimages libèrent des anticipés qui ont subi des influences diverses et qui sont axillés par des feuilles adultes. Les lots expérimentaux constitués par un type d'écimage sont composés d'une quarantaine de pousses sélectionnées sur une dizaine de ceps. Toutes les pousses d'un cep sont écimées de façon identique mais seules les pousses de grandeur et de diamètre moyens situés sur la baguette (taille Guyot) sont retenues pour les mesures de fertilité.

Après écimage, la fertilité des anticipés développés est évaluée à deux dates différentes :

- Dès la sortie des organes oppositifoliés, le nombre et la nature (grappe ou vrille) de ceux-ci sont notés. Tout organe oppositifolié avec des fleurs est considéré comme un organe fertile.

- Une deuxième mesure est faite lorsque les organes oppositifoliés sont plus développés afin d'apprécier leur chute ou leur maintien sur le rameau. La grosseur des grappes est alors estimée par un comptage des baies lorsque celles-ci mesurent 2 à $3 \mathrm{~mm}$ de diamètre.

Ces 2 mesures de fertilité permettent de tracer 2 courbes : 1 courbe de fertilité exprimée groupant tous les organes fertiles observables dès l'entrée en croissance des anticipés et 1 courbe de fertilité pratique ne faisant intervenir que les organes fertiles qui se maintiennent et fournissent des baies.

La fertilité des anticipés développés à la suite des différents écimages est comparée à la fertilité d'anticipés témoins portés par des sarments non écimés. Le lot témoin est composé d'une quarantaine de sarments.

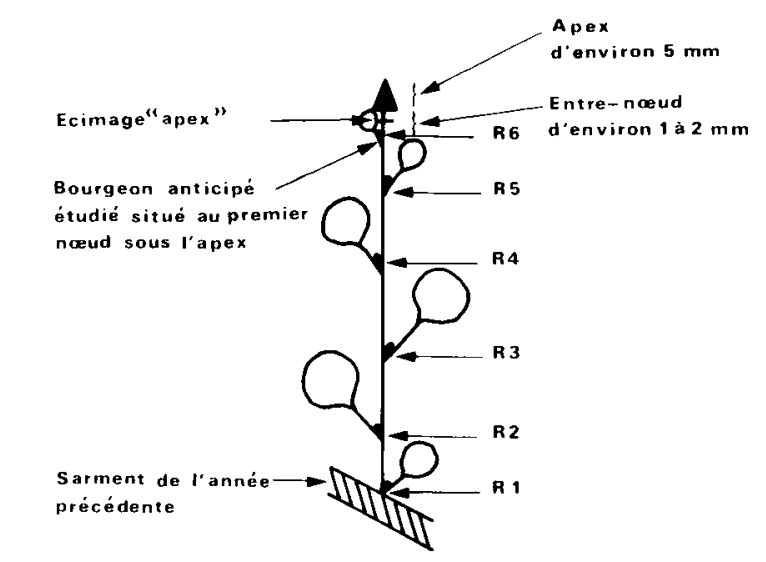

Figure 1

Exemple de l'écimage " apex » pratiqué au-dessus du næud 6, le 11 mai 1982.

Example of apex decapitation executed above node 6 on May 11th 1982.

\section{RÉSULTATS}

\section{A. Expression de la fertilité des anticipés sur sarments non écimés}

Sur le lot témoin représenté par des sarments de longueur équivalente, nous avons noté l'entrée en croissance des anticipés, l'importance de cette croissance et la fertilité pratique ; les résultats sont réunis au tableau 2. Quel que soit leur nœud d'insertion il y a toujours un certain pourcentage d'anticipés qui n'entrent pas en croissance, particulièrement à la base du sarment (rang 3) et ces anticipés n'extériorisent donc pas leur fertilité. Les anticipés qui entrent en croissance présentent une croissance très hétérogène et leur longueur moyenne est faible. Ces résultats sont en accord avec les analyses menées par HUGLIN (1958) et BOUARD (1966). Lorsque l'élongation de ces anticipés est voisine de $5 \mathrm{~cm}$, le plus souvent leur fertilité ne se manifeste pas car les organes oppositifoliés sont à peine dégagés. Lorsque leur croissance est plus grande, généralement les organes oppositifoliés tombent alors qu'ils sont encore très petits ( 2 à $5 \mathrm{~mm})$ et la fertilité pratique est infime ; la fertilité maximale est rencontrée au niveau des anticipés de rang 10 où elle n'est que de 0,3 grappe par rameau.

TABLEAU 2

Croissance et fertilité des anticipés sur sarments non écimés. Growth and fertility of the lateral shoots on non-decapitated shoots.

Anticipés Anticipés Anticipés Anticipés de rang 3 de rang 6 de rang 10 de rang 15

Pourcentage d'entrée

\begin{tabular}{lrrrr} 
en croissance & 40 & 83,3 & 96,6 & 96,5 \\
\hline $\begin{array}{l}\text { Longueur moyenne } \\
\text { (cm) }\end{array}$ & 4,9 & 9,2 & 18,1 & 10,7 \\
\hline
\end{tabular}

Fertilité pratique (nombre de grappes par rameau)

0

0,04

0,3 0 


\section{B. Expression de la fertilité des anticipés sur sarments écimés}

Lors des différents écimages, nous avons étudié la fertilité de l'anticipé situé immédiatement sous la décapitation: quels que soient l'importance et le niveau d'écimage, cet anticipé entre pratiquement toujours en croissance active. Les résultats sont détaillés pour les anticipés de rang 6 puis nous indiquons les similitudes et les différences observées pour les anticipés des autres rangs. Pour avoir une référence phénologique, nous signalons qu'en 1982 la floraison des inflorescences primordiales a débuté autour du 10 juin.

\section{Expression de la fertilité des anticipés de rang 6}

\section{a) Fertilité exprimée (fig. 2)}

La fertilité exprimée est d'abord faible, autour de 0,5 grappe par rameau pour des petits écimages; elle augmente progressivement jusqu'à un maximum de 1,9 grappes par rameau pour un écimage « apex $+11 \mathrm{~F}$ » effectué le 9 juin puis une légère baisse s'amorce pour les écimages tardifs. En effet, pour les écimages "apex +14 et $19 \mathrm{~F}$ », nous avons constaté que quelques organes oppositifoliés tombent sans que nous puissions déterminer leur nature : c'est ce phénomène qui est à l'origine de la diminution de fertilité. La fertilité globale se répartit essentiellement sur le $1^{\text {er }}$ organe oppositifolié où le nombre moyen de grappes tend rapidement vers 1 . La fertilité du $2^{\mathrm{e}}$ organe se manifeste plus tard (début juin) et semble se fixer autour de 0,7 grappe. Lorsque nous obtenons une fertilité maximale, il faut signaler que l'on observe quelquefois 3 grappes ou plus par rameau, ce qui correspond à un niveau de fertilité exprimée en nombre de grappes rarement atteint par les bourgeons latents principaux du cépage considéré.

\section{b) Fertilité pratique (fig. 3)}

La fertilité pratique est plus faible que la fertilité exprimée et la fertilité maximale est de 1,6 grappes par anticipé. Il existe toujours un certain pourcentage de chute des grappes mais ce pourcentage devient très important pour les traitements « apex +14 et $19 \mathrm{~F}$ »; la baisse de fertilité signalée à la figure 2 est accentuée et pour le dernier écimage la fertilité pratique des anticipés est presque nulle $(0,3$ grappe par rameau). Il semble que la chute des organes fertiles se produise aussi bien pour les $1^{\text {res }}$ grappes que pour les $2^{\text {es }}$ puisque le pourcentage de perte observé après le traitement «apex $+14 \mathrm{~F}$ » est de 32,5 pour le $1^{\text {er }}$ organe oppositifolié et de 28,5 pour le $2^{\text {e }}$, à la suite du traitement "apex $+19 \mathrm{~F}$ » il est respectivement de 88,7 et 85,9 . Certains auteurs ont rapporté des phénomènes semblables pour les grappes primordiales: BESSIS (1967) indique, sur des sarments de vigueur réduite, la possibilité d'un arrêt du développement des grappes et leur avortement, DURQUETY (1985) signale le dessèchement très précoce des ébauches inflorescentielles lors d'un mauvais débourrement des bourgeons latents.

A la suite des $1^{\text {ers }}$ écimages, les $1^{\text {res }}$ grappes ne portent que quelques baies, 10 à 20 en moyenne, réparties sur 1 ou 2 ramifications secondaires, puis le nombre de baies augmente en même temps que la fertilité jusqu'à un nombre moyen de 80 par grappe comptant 6 à 8 ramifications secondaires. La valeur maximale est rencontrée après un écimage au 14 juin pour une grappe de 230 baies. On peut rappeler que les grappes primordiales de Pinot portent classiquement entre 50 et 150 baies (BESSIS, 1965).

\section{Expression de la fertilité des anticipés de rang 10}

Les courbes de fertilité exprimée et de fertilité pratique sont données aux figures 4 et 5 , globalement le

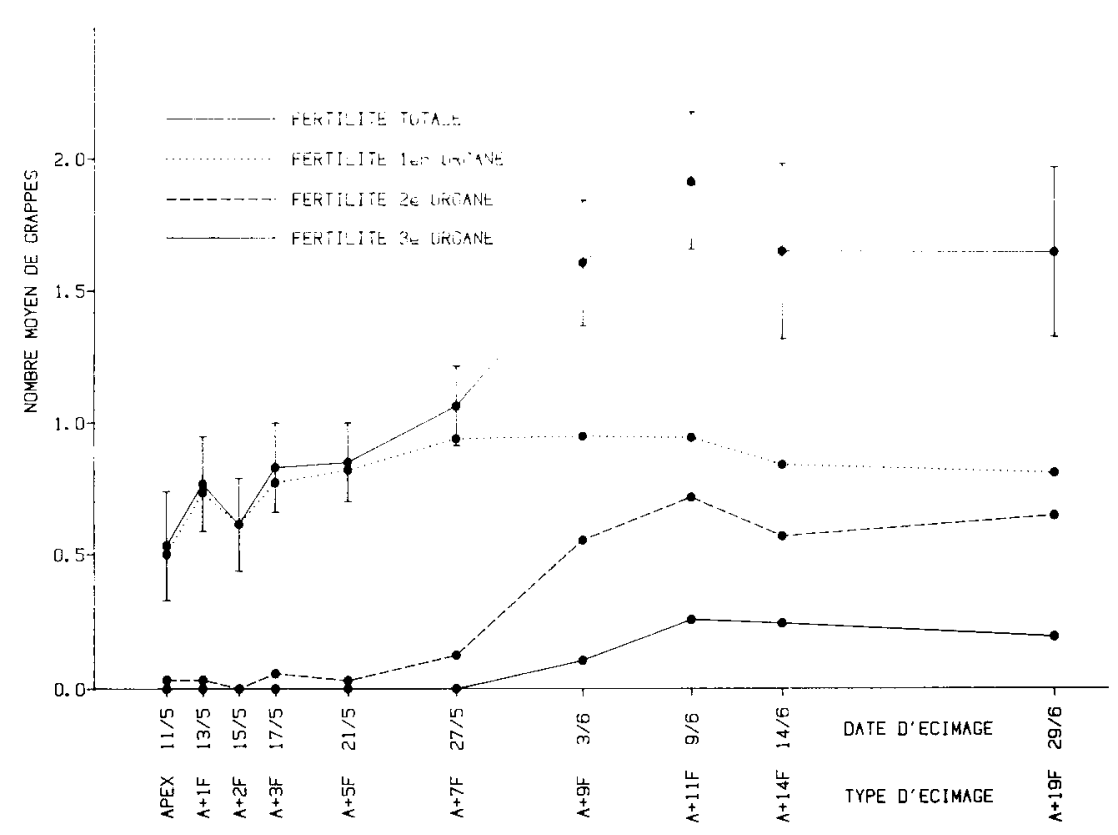

Figure 2

Fertilité exprimée des anticipés de rang 6 en fonction de la date d'écimage (intervalles de confiance pour un coefficient de sécurité de 95 p. 100).
Numbers of lateral shoots (order 6) forming inflorescences in relation to decapitation date $(95 \%$ confidence limits). 


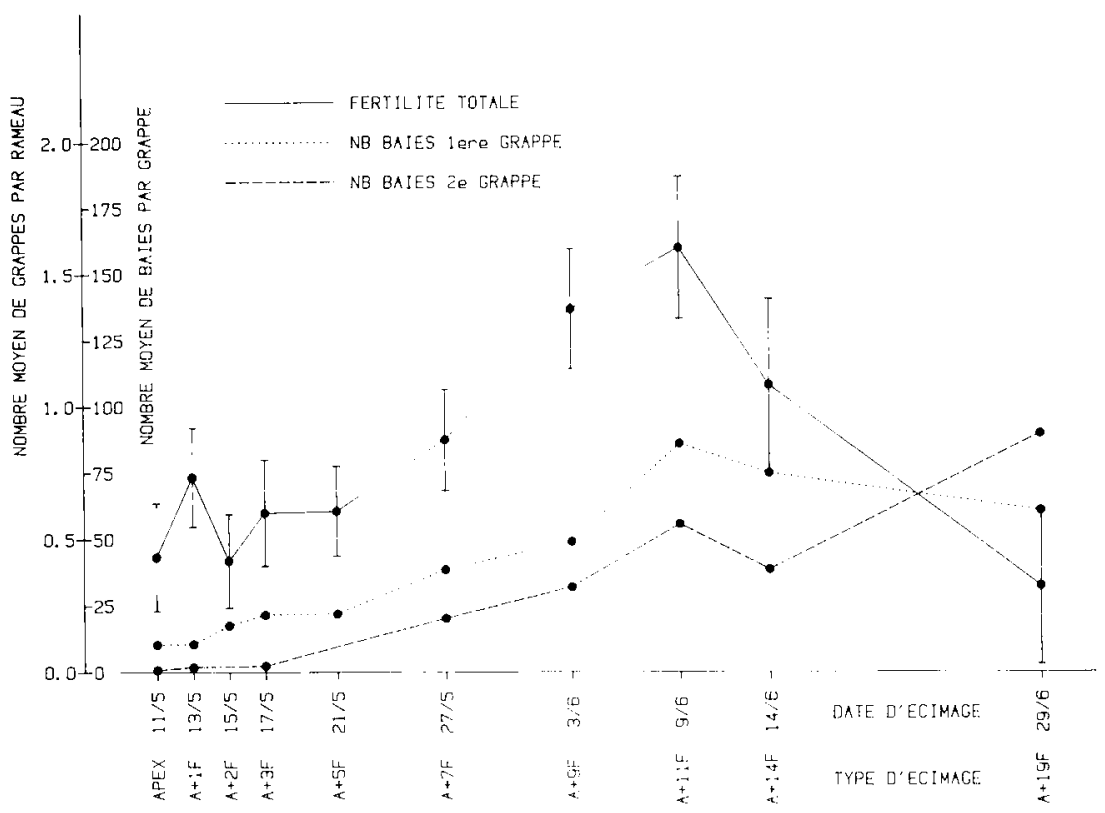

Figure 3

Fertilité pratique des anticipés de rang 6 en fonction de la date d'écimage.

Numbers of lateral shoots (order 6) in which inflorescences develop to maturity in relation to decapitation date.

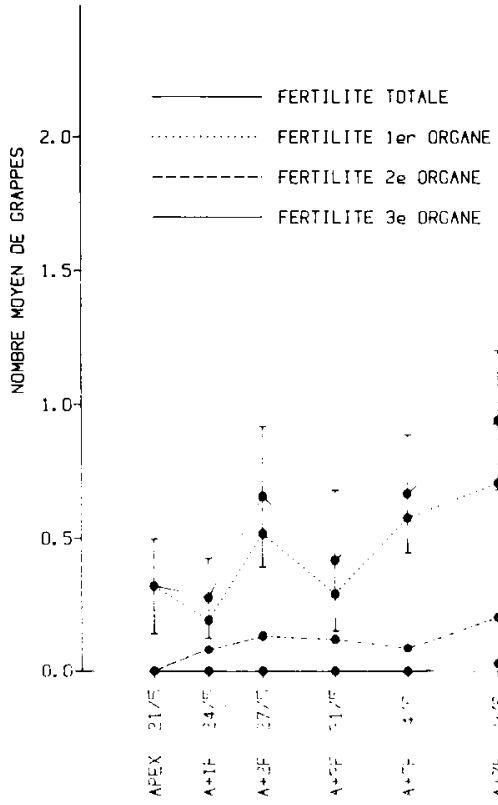

Figure 4

Fertilité exprimée des anticipés de rang 10 en fonction de la date d'écimage.

type de réponse est identique à celui des anticipés de rang 6 , à l'exception de 3 points :

- La fertilité exprimée est moins élevée puisqu'elle n'atteint que 1 grappe par rameau. La différence se répartit sur le $1^{\text {er }}$ et le $2^{\text {e }}$ organe oppositifolié où il existe un certain pourcentage de vrilles : au niveau du $1^{\text {er }}$ organe, après l'écimage «apex $+7 \mathrm{~F}$ » réalisé le 9 juin, on compte 70,6 p. 100 de grappes et 29,4 p. 100 de vrilles; au niveau du $2^{\mathrm{e}}$, après l'écimage " apex $+9 \mathrm{~F}$ » pratiqué le 14 juin, on dénombre 39,4 p. 100 de grappes et 60,6 p. 100 de vrilles. La baisse de fertilité observée aux écimages tardifs est également plus accentuée : à la suite de l'écimage «apex $+19 \mathrm{~F}$ » effectué le 8 juillet, la fertilité n'est que de 0,1 grappe par rameau ; les organes tombent lorsqu'ils mesurent 2 à $5 \mathrm{~mm}$ de longueur et nous ne pouvons déterminer leur nature.

- En fertilité pratique, la chute des grappes s'amplifie et la perte de fertilité est particulièrement prononcée pour les écimages « apex +14 et $19 \mathrm{~F}$ 》.

- L'importance des grappes est indiquée uniquement pour le $1^{\text {er }}$ organe oppositifolié car au niveau du $2^{\mathrm{e}}$ organe les grappes sont rares et les moyennes calculées sur un nombre réduit d'échantillons n'ont plus aucune signification. Les grappes sont plus petites et la valeur moyenne n'excède pas 50 baies par grappe. La valeur maximale est relevée pour un écimage réalisé le 18 juin avec une grappe de 182 baies. 


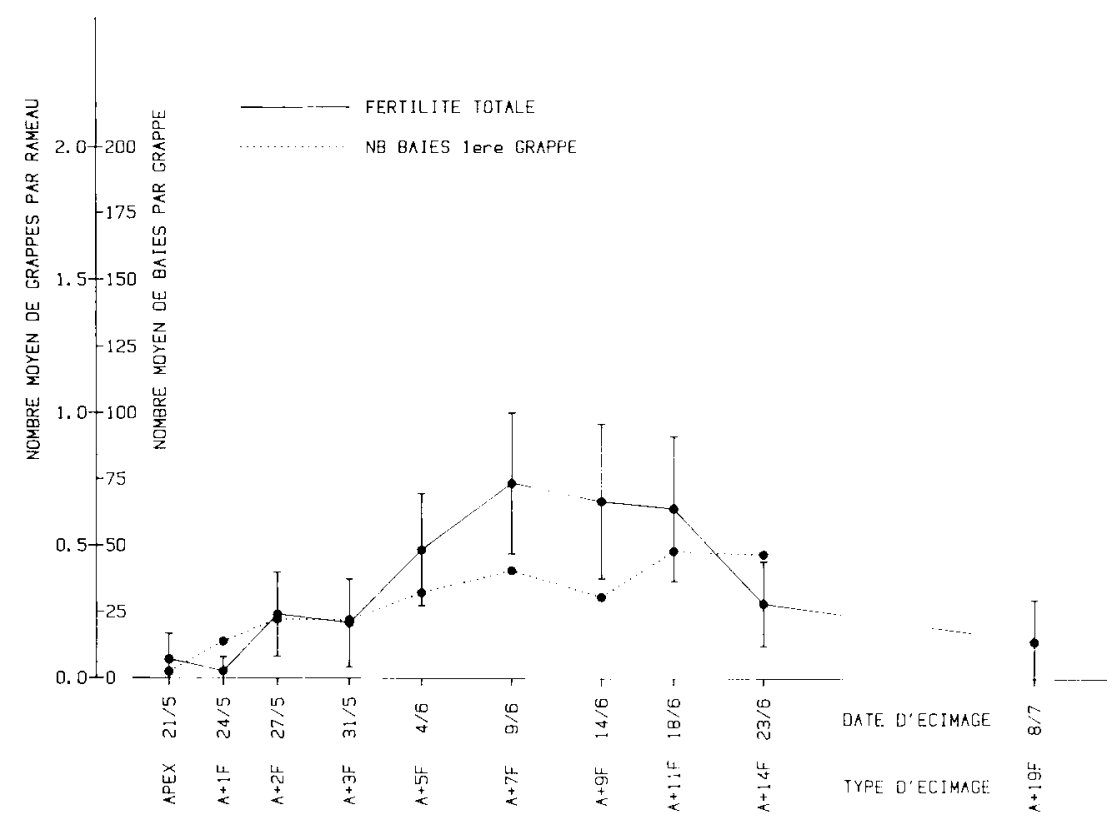

Figure 5

Fertilité pratique des anticipés de rang 10 en fonction de la date d'écimage.

Numbers of lateral shoots (order 10) in which inflorescences develop to maturity in relation to decapitation date.

\section{Expression de la fertilité des anticipés des autres rangs}

Par les méthodes d'écimage, nous avons étendu notre domaine d'expérimentation aux anticipés des rangs 3 et 15 . Afin de réaliser les expériences avec le même nombre de pieds par lot, nous avons travaillé sur une parcelle de Pinot différente mais avec une fertilité en grappes primordiales légèrement moins forte. Les expériences ont montré que le type de réponse observé pour les anticipés des rangs 6 et 10 est analogue pour tous les anticipés du sarment : la fertilité obtenue à la suite d'écimages petits et précoces est faible. Cette fertilité augmente régulièrement pour atteindre une valeur maximale après un écimage vers «apex $+9 \mathrm{~F}$ »; puis la fertilité diminue jusqu'à s'annuler lors des grands écimages comme «apex $+19 \mathrm{~F}$ ». Les fertilités maximales, fertilité exprimée et fertilité pratique, sont obtenues pour des anticipés de même âge (types d'écimage voisins) mais leur intensité varie avec le rang d'insertion des anticipés sur le sarment. Il semble que les anticipés de la base du sarment soient plus fertiles (1,3 grappes par rameau aux rangs 3 et 6) tandis que les anticipés formés plus tard sur le sarment ont une fertilité plus faible : 0,7 grappe par rameau au rang 10 et 0,5 au rang 15 .

En résumé, quel que soit leur rang d'insertion sur le sarment, les anticipés sont capables d'extérioriser une fertilité non négligeable mais celle-ci n'est obtenue et ne se maintient que dans des conditions précises. Si l'on considère le nombre de grappes, la fertilité maximale n'est parfois que légèrement inférieure à celle des sarments principaux. Par contre, la dimension des grappes reste plus petite : elle correspond à celle des grapillons qui apparaissent au vignoble après les divers rognages.

\section{CONCLUSION}

Le but de notre étude était de connaître le devenir des inflorescences formées dans les anticipés. Sur sarments non écimés, généralement la fertilité potentielle des anticipés ne s'exprime pas car la majorité d'entre eux n'entrent pas en croissance, lorsqu'ils entrent en croissance leur fertilité pratique est voisine de 0 . Sur sarments écimés, on peut distinguer 3 périodes dans l'expression de la fertilité : la fertilité est faible pour des anticipés développés après des petits écimages, elle augmente régulièrement jusqu'à une valeur maximale obtenue pour un écimage aux environs de « apex $+9 \mathrm{~F}$ », puis elle diminue pour s'annuler lors des grands écimages ( apex $+19 \mathrm{~F} »)$. Les facteurs qui interviennent à travers l'importance et l'époque d'écimage feront l'objet d'un second article.

Dans la pratique agronomique, l'objectif est parfois de faire produire du raisin par les anticipés (MAVRIKIOS, 1970 ; FAZINIC et al., 1976); il est difficile d'établir une comparaison entre les observations de ces auteurs et nos résultats car les indications sur les écimages réalisés sont vagues. Cependant, dans les conditions climatiques de nos régions, la production de grapillons est le plus souvent inutile voire nuisible à la qualité de la vendange. Il devient donc possible de se placer dans des conditions d'écimage entraînant une production minimale de ceux-ci.

Reçu le II décembre 1986. Accepté le 3 novembre 1987. 


\section{RÉFÉRENCES BIBLIOGRAPHIQUES}

Bessis R., 1965. Recherches sur la fertilité et les corrélations de croissance entre bourgeons chez la vigne (Vitis vinifera L.). Thèse, Dijon, 236 p.

Bessis R., 1967. A propos de l'existence et de la nature du filage des grappes chez la vigne. C. R. Acad. Agric. Fr., 1429-1436.

Bouard J., 1966. Recherches physiologiques sur la vigne et en particulier sur l'aôutement des sarments. Thèse, Bordeaux, $310 \mathrm{p}$.

Durquety P. M., 1985. Bassin de !'Adour en 84 : coulure et millerandage. Vititechnique, 85, 7-9.

Fazinic N., Briza K., Milosavljevic M., Lazic S., Cindric P., 1976.

Physiologie de la croissance et de la maturation des baies. Causes des variations des constituants principaux. Influence des operations en vert et des interventions chimiques. Bull. O.I.V., 545-546, 565574.

Huglin P., 1958. Recherches sur les bourgeons de la vigne, initiation florale et développement végétatif. Thèse, Strasbourg, $174 \mathrm{p}$.

Mavrikios Chr., 1970. A Chypre, les raisins sans pépins. Progr. Agr. et Vit., 22-23, 340-344 et 351-356.

Olivain C., Bessis R., 1987. L'organogenèse inflorescentielle dans les bourgeons anticipés de vigne (Vitis vinifera $\mathbf{L}$. cépage Pinot). Vitis, 26, 98-106. 